\title{
Super-robust nonadiabatic geometric quantum control
}

\author{
Bao-Jie Liu $\odot,{ }^{1}$ Yuan-Sheng Wang $\odot,{ }^{1,2}$ and Man-Hong Yung ${ }^{1,3,4,5, *}$ \\ ${ }^{1}$ Department of Physics, Southern University of Science and Technology, Shenzhen 518055, China \\ ${ }^{2}$ School of Physical Sciences, University of Science and Technology of China, Hefei 230026, China \\ ${ }^{3}$ Shenzhen Institute for Quantum Science and Engineering, Southern University of Science and Technology, Shenzhen 518055, China \\ ${ }^{4}$ Guangdong Provincial Key Laboratory of Quantum Science and Engineering, Southern University of Science \\ and Technology, Shenzhen 518055, China \\ ${ }^{5}$ Shenzhen Key Laboratory of Quantum Science and Engineering, Southern University of Science and Technology, Shenzhen 518055, China
}

(Received 18 March 2021; accepted 7 September 2021; published 16 September 2021)

\begin{abstract}
Nonadiabatic geometric quantum computation (NGQC) and nonadiabatic holonomic quantum computation (NHQC) have been proposed to reduce the run time of geometric quantum gates. However, in terms of robustness against experimental control errors, the existing NGQC and NHQC scenarios have no advantage over standard dynamical gates in most cases. Here, we give the reasons why nonadiabatic geometric gates are sensitive to the control errors and, further, we propose a scheme of super-robust nonadiabatic geometric quantum control, in which the super-robust condition can guarantee both high speed and robustness of the geometric gate. To illustrate the working mechanism of super-robust geometric quantum gates, we give two simple examples of SR-NGQC and SR-NHQC for two- and three-level quantum systems, respectively. Theoretical and numerical results with the experimental parameters indicate that our scheme can significantly improve the gate performance compared to the previous NGQC, NHQC, and standard dynamical schemes. Super-robust geometric quantum computation can be applied to various physical platforms such as superconducting qubits, quantum dots, and trapped ions. All of these sufficiently show that our scheme provides a promising way towards robust geometric quantum computation.
\end{abstract}

DOI: 10.1103/PhysRevResearch.3.L032066

Introduction. Realizing high-fidelity and fault-tolerant quantum gates is very essential for quantum information processing since control errors and environment-induced noises are ubiquitous in operating real quantum devices. Geometric quantum computation (GQC) utilizes a unique property that the time-dependent quantum state would accumulate an Abelian geometric phase [1,2] or non-Abelian holonomy [3-5] under a cyclic quantum evolution. The geometric phase and holonomy depend only on the global properties of the evolution trajectories. Consequently, geometric quantum gates are robust against local disturbances during the evolution [6-11]. More specifically, geometric quantum computation can be divided into Abelian GQC and holonomic quantum computation (HQC) depending on whether the geometric phase is a real number [1] or a matrix [4] (non-Abelian holonomy).

Early applications of GQC are dependent on adiabatic quantum evolutions to suppress transitions between different instantaneous eigenstates of the Hamiltonian [12-16]. Adiabatic GQC has been experimentally verified as a

\footnotetext{
*yung@ sustech.edu.cn

Published by the American Physical Society under the terms of the Creative Commons Attribution 4.0 International license. Further distribution of this work must maintain attribution to the author(s) and the published article's title, journal citation, and DOI.
}

noise-resilient scenario against fluctuations of control parameters [13,14]. However, adiabatic quantum dynamics implies lengthy gate time and thus long exposure time to the environment-induced decoherence. To overcome such a problem, nonadiabatic geometric quantum computation (NGQC) [17-23] and nonadiabatic holonomic quantum computation (NHQC) [24-36] based on a nonadiabatic Abelian and non-Abelian geometric phase [2,4], respectively, have been proposed to reduce the run times of geometric quantum gates. Recently, nonadiabatic geometric gates have been experimentally demonstrated in different physical platforms, including superconducting qubits [37-45], nuclear magnetic resonance (NMR) [46-48], and nitrogen-vacancy centers in diamond [49-54]. However, in terms of robustness against experimental errors, the existing NGQC and NHQC gates have no sufficient preponderance over standard dynamical gates in most cases [19,34-36]. Therefore, it is natural to ask (i) why the existing nonadiabatic geometric gates lack robustness against the control errors and (ii) how to maintain both the speed and the robustness of geometric gates.

In this Letter, we give clear answers for the above two important issues using the super-robust condition proposed here. And on that basis, we demonstrate a class of super-robust nonadiabatic geometric gates in which robustness against the control errors is ensured by a super-robust control condition. We implement our schemes in two- and three-level systems, respectively, to realize super-robust Abelian (non-Abelian) nonadiabatic geometric (holonomic) quantum gates, called 
SR-NGQC (SR-NHQC). Using the experimental parameters, the numerical results indicate that our scheme can significantly improve the gate performance compared to the existing NGQC, NHQC, and standard dynamical schemes, which are in good agreement with the theoretical results. In addition, these super-robust geometric quantum computations can be easily applied to various physical platforms.

General framework of nonadiabatic geometric quantum control. Let us start with a nondegenerate quantum system described by $(M+N)$-dimensional Hilbert space, with its evolution governed by the Hamiltonian $H(t)$. For any complete set of basis vectors $\left\{\left|\psi_{k}(0)\right\rangle\right\}_{k=1}^{M+N}$ at $t=0$, the time-evolution operator can be written as $U(t)=\mathcal{T} e^{-i \int_{0}^{t} H\left(t^{\prime}\right) d t^{\prime}}=\sum_{m}\left|\psi_{m}(t)\right\rangle\left\langle\psi_{m}(0)\right|$, where the timedependent state, $\left|\psi_{m}(t)\right\rangle=\mathcal{T} e^{-i \int_{0}^{t} H\left(t^{\prime}\right) d t^{\prime}}\left|\psi_{m}(0)\right\rangle$, follows the Schrödinger equation. Here, we choose a different set of timedependent auxiliary basis states $\left\{\left|\mu_{k}(t)\right\rangle\right\}_{k=1}^{M+N}$, which makes the Hamiltonian $H(t)$ satisfy the decomposition condition. The decomposition condition ensures that the operations in the noncomputational basis $\left\{\left|v_{k}(t)\right\rangle\right\}_{k=M+1}^{M+N}$ are completely irrelevant for the geometric operations in the computational subspace $\left\{\left|\mu_{k}(t)\right\rangle\right\}_{k=1}^{M}$, which is given by

$$
\begin{aligned}
H_{R}(t) & =V^{+}(t)\left[H(t)-i \partial_{t}\right] V(t) \\
& =H_{C}(t) \oplus H_{N}(t),
\end{aligned}
$$

where $V(t) \equiv \sum_{k}\left|\mu_{k}(t)\right\rangle\left\langle\mu_{k}(0)\right|, H_{C}(t) \equiv \sum_{m, k=1}^{M}\left[\left\langle\mu_{m}\right| H(t)-\right.$ $\left.i \partial_{t}\left|\mu_{k}\right\rangle\right] \Pi_{m k}(0)$ is a Hamiltonian acting on the $M$-dimensional computational subspace with $\Pi_{m k}(0) \equiv\left|\mu_{m}(0)\right\rangle\left\langle\mu_{k}(0)\right|$, and $H_{N}(t)=\sum_{m, k=M+1}^{M+N}\left[\left\langle\mu_{m}\left|H(t)-i \partial_{t}\right| \mu_{k}\right\rangle\right] \Pi_{m k}(0)$ is Hamiltonian acting on the noncomputational subspace, as shown in Fig. 1(a).

Now, we explain how to construct a nonadiabatic geometric gate in the computational subspace. With the help of $\left\{\left|\mu_{k}(t)\right\rangle\right\}_{k=1}^{M},\left|\psi_{m}(t)\right\rangle$ can be expressed as $\left|\psi_{m}(t)\right\rangle=\sum_{l=1}^{M} C_{l m}(t)\left|\mu_{l}(t)\right\rangle$, and the time-evolution operator in the computational subspace becomes $U_{C}(t)=\sum_{l, m=1}^{M} C_{l m}(t)\left|\mu_{l}(t)\right\rangle\left\langle\mu_{m}(0)\right|$. After a cyclic evolution, we obtain the final time-evolution operator [24], $\quad U_{C}(\tau)=\sum_{m, k=1}^{M}\left[\mathcal{T} e^{i \int_{0}^{\tau} \mathbf{A}(t)+\mathbf{K}(t) d t}\right]_{m k} \Pi_{m k}(0), \quad$ where $\mathcal{T}$ is the time-ordering operator, $\mathbf{A}_{l m} \equiv i\left\langle\mu_{l}(t)\left|\partial_{t}\right| \mu_{m}(t)\right\rangle$ is the matrix-valued connection one-form, and $\mathbf{K}_{l m}(t) \equiv$ $-\left\langle\mu_{l}(t)|H(t)| \mu_{m}(t)\right\rangle$ is the dynamical part.

To meet the decomposition condition given by Eq. (1), one possible set of the auxiliary state $\left|\mu_{k}(t)\right\rangle$ is found to be proportional to the time-evolution states $\left|\psi_{k}(t)\right\rangle$, i.e., $\left|\mu_{k}(t)\right\rangle=$ $e^{i f_{k}(t)}\left|\psi_{k}(t)\right\rangle$. Then, we obtain the nondiagonal parts of $\mathbf{A}$ and $\mathbf{K}$, which satisfy the relation

$$
\mathbf{A}_{l m}(t)=-\mathbf{K}_{l m}(t)=e^{-i \delta_{k m}(t)}\left\langle\psi_{l}(t)|H(t)| \psi_{m}(t)\right\rangle,
$$

where $\delta_{k m}(t)=f_{k}(t)-f_{m}(t)$. Using the spin-echo technique [12] or the pulse-shaping method [24], the accumulated dynamical phases can be erased, i.e., $\int_{0}^{\tau} K_{m m}(t) d t=0, m=$ $1, \ldots, M$. In this way, we will obtain

$$
U_{C}(\tau)=\sum_{m=1}^{M} e^{i \int_{0}^{\tau} \mathbf{A}_{m m}(t) d t} \Pi_{m m}(0),
$$

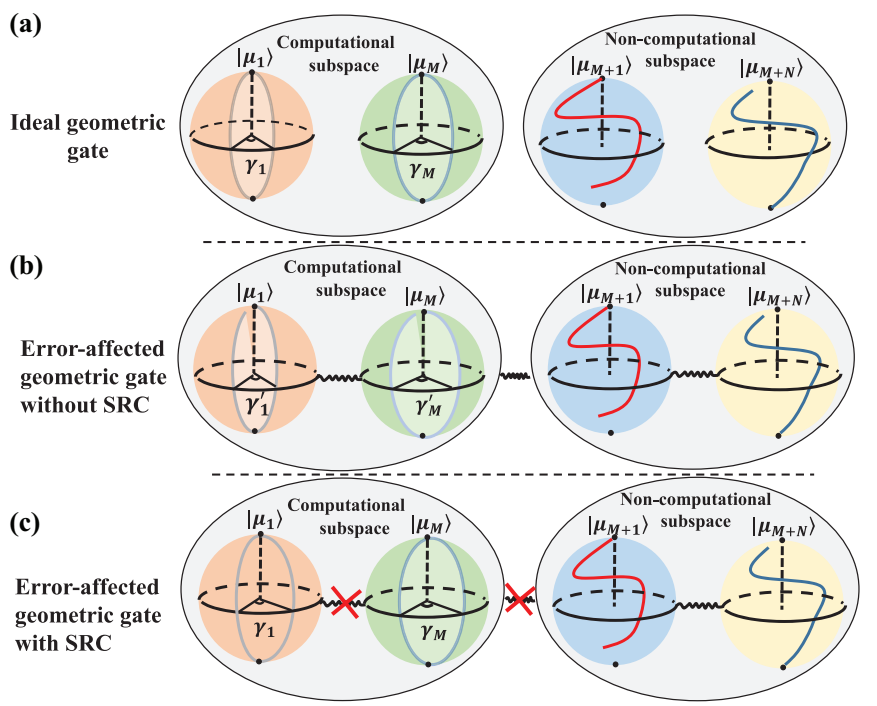

FIG. 1. The illustration of our proposed implementation. (a) Conceptual explanation for the ideal nonadiabatic geometric quantum gates in a $(M+N)$-dimensional Hilbert space. The evolution state $\left|\mu_{k}(t)\right\rangle$ acquired a pure nonadiabatic geometric phase $\gamma_{k}$ under a cyclic evolution in computational subspace to construct geometric gates. Meanwhile, the state evolution in a noncomputational basis is completely irrelevant for geometric operations in the Hilbert space. (b) Without super-robust condition (SRC) protection, the fidelity of the nonadiabatic geometric quantum gate with the presence of global control errors is limited because of the unwanted couplings of the time-dependent auxiliary states in the computational and noncomputational subspace. (c) With SRC protection, the effects of the above couplings can be greatly suppressed.

which is a nonadiabatic geometric quantum gate in the computational subspace $\left\{\left|\mu_{m}(t)\right\rangle\right\}_{m=1}^{M}$.

Super-robust condition. Now, we consider the effect of control error on the quantum evolution. The ideal Hamiltonian $H(t)$ then becomes

$$
H^{\prime}(t)=H(t)+\beta V(t),
$$

where we assume that $\beta$ is a small constant, i.e., $|\beta| \ll 1$, which corresponds to a quasistatic noise $[55,56]$. Here, $V(t)$ is the noise Hamiltonian that destroys the ideal dynamics, which can be regarded as a perturbation [57]. Under this assumption, the decomposition condition given by Eq. (1) is broken. In other words, the rotating Hamiltonian becomes $H_{R}^{\prime}(t)=$ $\sum_{m=1}^{M+N} \mathbf{A}_{m m}(t) \Pi_{m m}(0)+\beta \sum_{m \neq k}^{M+N} \mathbf{K}_{m k}(t) \Pi_{m k}(0)$. Then, we obtain the evolution operator as $U_{E}(\tau)=\mathcal{T} e^{-i \int_{0}^{\tau} H_{R}^{\prime}(t) d t}$. In general, to analytically solve this equation is difficult due to the time-ordering operator.

Here, we use the Magnus expansion [57-59] to perturbatively process the evolution operator $U_{E}(\tau)$. Before that, we transform to the interaction picture by defining $U_{I}(t)=$ $\sum_{m=1}^{M+N} e^{-i \int_{0}^{t} \mathbf{A}_{m m}\left(t^{\prime}\right) d t^{\prime}} \Pi_{m m}(0)$, and the transformed Hamiltonian is $H_{I R}^{\prime}(t)=\beta U_{I}^{+}(t) \sum_{m \neq k}^{M+N} \mathbf{K}_{m k}(t) \Pi_{m k}(0) U_{I}(t)$. The corresponding evolution operator in the interaction frame is given by

$$
U_{I E}(\tau)=\mathcal{T} e^{-i \int_{0}^{\tau} H_{I R}^{\prime}(t) d t}
$$


Applying the Magnus expansion to Eq. (5), we have

$$
U_{I E}(\tau)=e^{\sum_{k=1}^{\infty} \Lambda_{k}(\tau)},
$$

where $\Lambda_{k}$ denotes the terms of the Magnus expansion, and the first two terms of the series are given by (see, e.g., [57-59])

$$
\begin{aligned}
& \Lambda_{1}(t)=-i \int_{0}^{t} H_{I R}^{\prime}\left(t_{1}\right) d t_{1}, \\
& \Lambda_{2}(t)=\frac{1}{2} \int_{0}^{t} d t_{1} \int_{0}^{t_{1}} d t_{2}\left[H_{I R}^{\prime}\left(t_{1}\right), H_{I R}^{\prime}\left(t_{2}\right)\right] .
\end{aligned}
$$

Expanding Eq. (6) in powers of $\beta$ and using Eq. (7), we obtain

$$
U^{\prime}(\tau, 0)=U(\tau, 0)\left[I-i \beta D(\tau)-\frac{\beta^{2}}{2} G(\tau)+O\left(\beta^{3}\right)\right],
$$

where the element of matrix $D_{k m}(t) \equiv$ $\int_{0}^{t}\left\langle\psi_{k}\left(t^{\prime}\right)\left|V\left(t^{\prime}\right)\right| \psi_{m}\left(t^{\prime}\right)\right\rangle d t^{\prime}$ and $G(\tau) \equiv \int_{0}^{\tau} d t[\dot{D}(t), D(t)]+$ $D^{2}(\tau)$. Consequently, the geometric gate in Eq. (3) under the control error becomes $U_{C}^{\prime}(\tau, 0)=$ $\sum_{m=1}^{M}\left|\psi_{m}^{\prime}(\tau)\right\rangle\left\langle\psi_{m}(0)\right|=\sum_{m=1}^{M} N_{r m} U^{\prime}(\tau, 0) \Pi_{m m}(0)$, where $N_{r m}=1 / \sqrt{1+\beta^{2} \sum_{k=1}^{M+N}\left|D_{k m}\right|^{2}}$ is the state normalized coefficient. To further evaluate the performance of the quantum operation caused by the control error, the gate fidelity $[60,61]$ is taken by

$$
\begin{aligned}
F & =\frac{1}{M}\left|\operatorname{Tr}\left(U_{C}^{\prime} U_{C}^{\dagger}\right)\right| \\
& \approx 1-\frac{\beta^{2}}{2 M} \sum_{m=1}^{M} \sum_{k=1}^{M+N}\left|D_{k m}\right|^{2}-\mathcal{O}\left(\beta^{4}\right) .
\end{aligned}
$$

Consequently, we can achieve super-robust gates with the fourth-order error dependence against the control error, as long as the following condition is satisfied:

$$
D_{k m} \equiv \int_{0}^{\tau}\left\langle\psi_{k}(t)|V(t)| \psi_{m}(t)\right\rangle d t=0,
$$

where $k=1, \ldots, M+N$ and $m=1,2, \ldots, M$.

As a demonstration of Eq. (10), we consider that the control error is global with $V(t)=H(t)$. In this case, the super-robust condition can be expressed by

$$
D_{k m}^{\prime}=\int_{0}^{\tau}\left\langle\psi_{k}(t)|H(t)| \psi_{m}(t)\right\rangle d t=0 .
$$

In this way, the effects of global control errors can be greatly suppressed. Here, we further illustrate the geometric meaning of Eq. (11): $D_{m m}^{\prime}=0$ with $k=m$ erases the accumulated dynamical phases, and $D_{k m}=\int_{0}^{\tau} e^{i \delta_{k m}} \mathbf{A}_{k m} d t=0$ with $k \neq m$ suppresses both couplings between the time-dependent auxiliary states $\left|\mu_{m}(t)\right\rangle$ and $\left|\mu_{n}(t)\right\rangle$ in the computational subspace and noncomputational subspace under the control errors, as shown in Figs. 1(b) and 1(c).

Note that the key difference between the previous NGQC and NHQC schemes and the super-robust condition-based scheme proposed here is that the Hamiltonians have different constraints. In the NGQC case, the Hamiltonian is only required to satisfy the constraint $D_{m n}^{\prime}=0$. The constraint of NHQC is set as $D_{m n}^{\prime}=0$ with $m, n=1, \ldots, M$. We can clearly see that the constraints of NGQC and NHQC are a necessary but not sufficient condition of super-robust gates. (a)

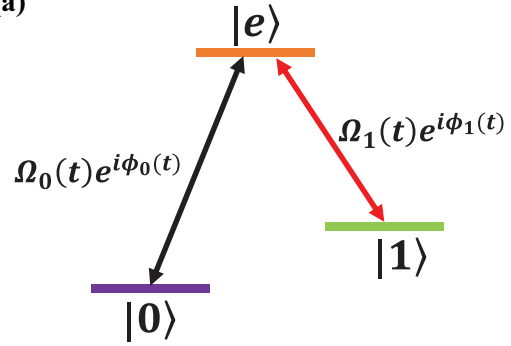

(b)

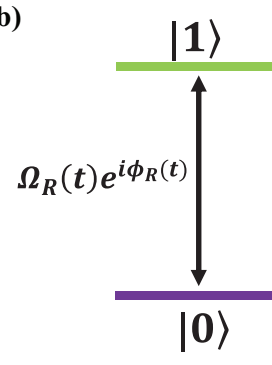

FIG. 2. The level structure and coupling configuration for the construction of SR-NHQC and SR-NGQC gates. (a) The driven pulses with amplitudes $\Omega_{0}$ and $\Omega_{1}$ resonantly couple $|0\rangle$ and $|1\rangle$ to $|e\rangle$ with the phases $\phi_{0}$ and $\phi_{1}$, respectively. (b) The driven pulse with amplitudes $\Omega_{R}$ resonantly couples $|0\rangle$ and to $|1\rangle$ with the timeindependent phase $\phi_{R}$.

Therefore, we can explain why some previous NGQC and NHQC lack robustness to the global control errors.

In addition, we can further explain the origin of the robustness of adiabatic GQC [12-14] against the control errors. The adiabatic condition [2,62] for a nondegenerate system is $\left|A_{k m}\right|=\left|\frac{\left\langle e_{k}\left|\partial_{t} H\right| e_{m}\right\rangle}{E_{k}-E_{m}}\right| \ll 1$ for $k \neq m$, where $\left\{E_{k}(t)\right\}$ and $\left|e_{k}(t)\right\rangle$ denote the instantaneous eigenvalues and eigenvectors of $H(t)$. In this case, we consider the auxiliary basis states $\left|\mu_{k}(t)\right\rangle$ to be identical to the eigenvectors $\left|e_{k}(t)\right\rangle$. The adiabatic condition ensures that the conditions of Eqs. (1) and (11) are met. Consequently, we can verify that the fidelity of the adiabatic GQC has, at least, a fourth-order error dependence against global control errors, using Eq. (9). In the following, to illustrate the working mechanism of super-robust geometric gates, we give two simple examples of SR-NGQC and SRNHQC for two- and three-level quantum systems, respectively

Example 1: SR-NHQC. The main idea of the previous NHQC is to generate a nonadiabatic non-Abelian geometric gate in a three-level system, as shown in Fig. 2(a). Under the rotating-wave approximation, the system Hamiltonian is given by $H(t)=\sum_{i=0}^{1} \frac{1}{2}\left[\Omega_{i}(t) e^{i \phi_{i}}|i\rangle\langle e|+\right.$ H.c. $]$. We define a bright state, $|b\rangle \equiv \sin \left(\frac{\theta}{2}\right) e^{i \phi}|0\rangle+\cos \left(\frac{\theta}{2}\right)|1\rangle$, where $\phi \equiv$ $\phi_{0}(t)-\phi_{1}(t)$ and $\tan (\theta / 2) \equiv \Omega_{0}(t) / \Omega_{1}(t)$. We shall keep $\theta$ and $\phi$, and hence $|b\rangle$, to be time independent. The Hamiltonian $H(t)$ can then be rewritten as $H(t)=\frac{1}{2}\left(\Omega(t) e^{i \phi_{1}(t)}|b\rangle\langle e|+\right.$ H.c. ), where $\Omega(t) \equiv \sqrt{\Omega_{0}(t)^{2}+\Omega_{1}(t)^{2}}$ is the Rabi frequency of $H(t)$.

Here, we choose the time-dependent auxiliary basis states as $\left|\mu_{1}(t)\right\rangle=|d\rangle, \quad\left|\mu_{2}(t)\right\rangle=\cos \frac{\alpha(t)}{2}|b\rangle-i \sin \frac{\alpha(t)}{2} e^{-i \phi_{1}(t)}|e\rangle$, and $\left|\mu_{3}(t)\right\rangle=-i \sin \frac{\alpha(t)}{2} e^{i \phi_{1}(t)}|b\rangle+\cos \frac{\alpha(t)}{2}|e\rangle$ with $\alpha(t)=$ $\int_{0}^{t} \Omega\left(t^{\prime}\right) d t^{\prime}$ (see the Supplemental Material [63] for details). Therefore, the rotating Hamiltonian given by Eq. (1) in the auxiliary basis states is given by $H_{R}(t)=\frac{1-\cos \alpha}{2} \dot{\phi}_{1}|b\rangle\langle b| \oplus$ $\frac{\cos \alpha-1}{2} \dot{\phi}_{1}|e\rangle\langle e|$. The corresponding computational Hamiltonian and noncomputational Hamiltonian are given by $H_{C}(t)=$ $\frac{1-\cos \alpha}{2} \dot{\phi}_{1}|b\rangle\langle b|+0| d\rangle\langle d|$ and $H_{N}(t)=\frac{\cos \alpha-1}{2} \dot{\phi}_{1}|e\rangle\langle e|$, respectively. Since the parallel transport conditions of the previous NHQC [17-23,37-39], i.e., $\left\langle\psi_{m}(t)|H(t)| \psi_{n}(t)\right\rangle=$ $0(n, m=1,2)$, are satisfied, the super-robust condition given 
TABLE I. The robustness comparison with the NOT gate of the standard dynamical gate (DG), nonadiabatic geometric gate, holonomic gate, and super-robust geometric quantum gates.

\begin{tabular}{lcccc}
\hline \hline Types & System level & Gate time & Fidelity & References \\
\hline DG & 2 & $\pi / \Omega_{0}$ & $1-\beta^{2} \pi^{2} / 8$ & \\
NGQC & 2 & $2 \pi / \Omega_{0}$ & $1-\beta^{2} \pi^{2} / 8$ & {$[17-23,37-39]$} \\
SR-NGQC & 2 & $3 \pi / \Omega_{0}$ & $1-\mathcal{O}\left(\beta^{4}\right)$ & This work \\
NHQC & 3 & $2 \pi / \Omega_{0}$ & $1-\beta^{2} \pi^{2} / 3$ & {$[25-36,42-54]$} \\
SR-NHQC & 3 & $4 \pi / \Omega_{0}$ & $1-\mathcal{O}\left(\beta^{4}\right)$ & This work \\
\hline \hline
\end{tabular}

by Eq. (11) can be reduced to

$$
D_{23}^{\prime}=\int_{0}^{\tau} \frac{\dot{\alpha}}{2} \exp \left(-i \int_{0}^{t} \dot{\phi}_{1} / \cos \alpha d t^{\prime}\right) d t=0 .
$$

Consequently, we obtain the following unitary transformation matrix in the basis states: $\left\{\left|\mu_{1}(0)\right\rangle,\left|\mu_{2}(0)\right\rangle\right\}$, i.e., $U_{C}(\tau)=e^{i \gamma_{g}}|b\rangle\langle b|+| d\rangle\langle d|$, where $\gamma_{g}=\int_{0}^{\tau} \frac{\cos \alpha-1}{2} \dot{\phi}_{1} d t=$ $\frac{1}{2} \int_{\phi_{1}(0)}^{\phi_{1}(\tau)} \int_{\alpha(0)}^{\alpha(\tau)} \sin \alpha d \alpha d \phi_{1}$, which shows that the geometric phase $\gamma_{g}$ exactly equals half of the solid angle. Note that the nonadiabatic holonomic gate can be spanned by the logical basis $\{|0\rangle,|1\rangle\}$, i.e.,

$$
U\left(\gamma_{g}, \theta, \phi\right)=e^{i \frac{\gamma_{g}}{2} \mathbf{n} \cdot \sigma},
$$

where $\boldsymbol{n}=(\sin \theta \cos \phi, \sin \theta \sin \phi, \cos \theta)$, and $\boldsymbol{\sigma}$ are the Pauli matrices. Equation (13) describes a rotational operation around the $\boldsymbol{n}$ axis by a $\gamma_{g}$ angle, ignoring a global phase factor $e^{-i \frac{\gamma_{g}}{2}}$. Since both $\boldsymbol{n}$ and $\gamma_{g}$ can take any value, Eq. (13) denotes a set of universal single-qubit gates in the qubit subspace.

Example 2: SR-NGQC. As another example, one can also apply our scheme to a two-level system, as shown in Fig. 2(b). The system Hamiltonian can be written as $H_{1}(t)=$ $\frac{\Omega_{R}(t)}{2} e^{i \phi_{R}(t)}|0\rangle\langle 1|+$ H.c. The time-dependent auxiliary basis states are taken by $\left|\zeta_{1}(t)\right\rangle=\cos \frac{\alpha_{R}(t)}{2}|0\rangle-i \sin \frac{\alpha_{R}(t)}{2} e^{-i \phi_{R}(t)}|1\rangle$ and $\left|\zeta_{2}(t)\right\rangle=-i \sin \frac{\alpha_{R}(t)}{2} e^{i \phi_{R}(t)}|0\rangle+\cos \frac{\alpha_{R}(t)}{2}|1\rangle$ with $\alpha_{R}(t)=$ $\int_{0}^{t} \Omega_{R}\left(t^{\prime}\right) d t^{\prime}+\alpha_{R}(0)$. Under these settings, the condition of the previous NGQC [17-23,37-39], i.e., $D_{m m}^{\prime}=$ $\int_{0}^{\tau} \mathbf{K}_{m m}(t) d t=0, m=1,2$, is satisfied. Thus, the superrobust condition given by Eq. (11) for NGQC becomes

$$
D_{12}^{\prime}=\int_{0}^{\tau} \frac{\dot{\alpha}_{R}}{2} \exp \left(-i \int_{0}^{t} \dot{\phi}_{R} / \cos \alpha_{R} d t^{\prime}\right) d t=0 .
$$

Similar to the SR-based NHQC case, we can also obtain the universal single-qubit gates in Eq. (13), i.e., $U\left[\gamma_{R}, \alpha_{R}(0), \phi_{R}(0)\right]$, with the geometric phase $\gamma_{R}=\int_{0}^{\tau} \dot{\phi}_{R}\left(\cos \alpha_{R}-1\right) / 2 d t$.

Numerical simulations. To investigate the noise-resilient feature of super-robust geometric gates against the global control error, we take the NOT gate as a typical example to compare the performances of the SR-NHQC and SR-NGQC approaches with that of the related standard dynamical gate (DG), NGQC, and NHQC approaches. Before that, we choose time-independent Rabi frequencies of all the gates and keep the same as $\Omega(t)=\Omega_{R}(t)=\Omega_{0}$; the corresponding gate times are sketched in Table I (see the Supplemental Material [63] for details). Here, we assume the relative pulse deviation $\beta$ of the driven pulse to vary in the range of $\beta \in[-0.1,0.1]$. As
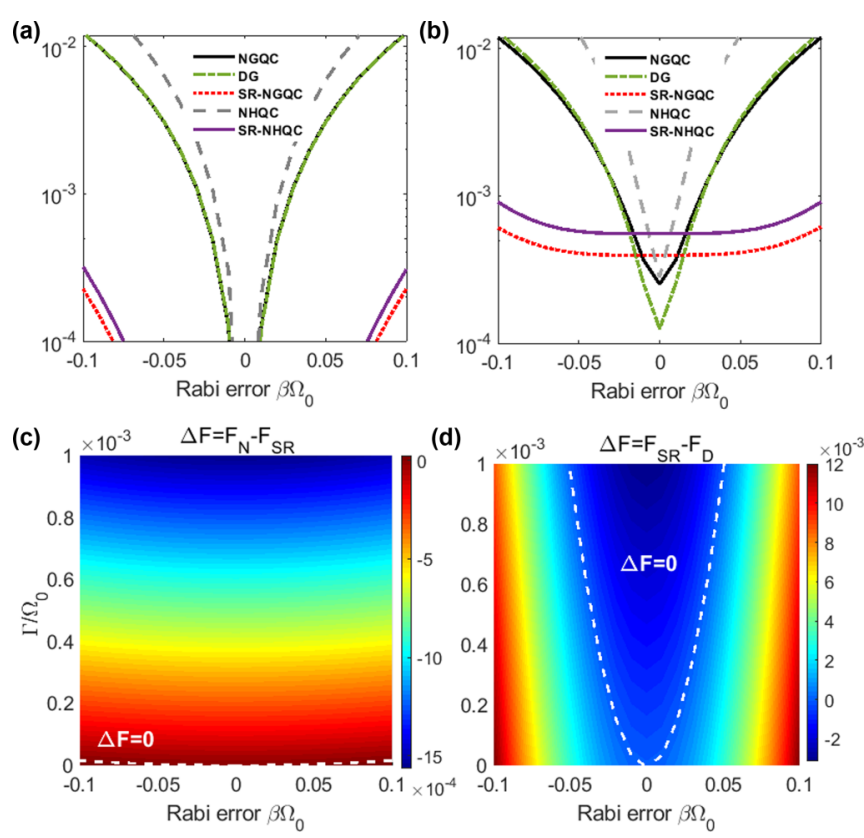

FIG. 3. Numerical robustness comparison of the NOT gate with various approaches. The NOT gate infidelities $1-F$ of DG, NGQC, SR-NGQC, NHQC, and SR-NHQC are set as a function of the control error of the Rabi frequency, i.e., the relative pulse deviation $\beta$ (a) without and (b) with the decoherence. The NOT gate fidelities' difference, (d) $\Delta F=F_{G}-F_{D}$ and (c) $\left(\Delta F=F_{N}-F_{D}\right)$, between the SR-NGQC (NGQC) and DG are set as the decoherence parameter $\Gamma$ and the relative pulse deviation $\beta$.

shown in Fig. 3(a), the SR-NHQC and SR-NGQC are always more robust than the DG, NHQC, and NGQC gates without the consideration of decoherence. The numerical results are in good agreement with the theoretical results, as shown in Table I.

In fact, the decoherence process is unavoidable. To evaluate the performance of the gates with the consideration of the influence of decoherence, the Lindblad master equation [64] is used here. To be more eloquent, we set the parameters from the current experiments [65-67]: the decay and dephasing rates of the qubit are taken as $\Gamma_{1}=\Gamma_{2}=\Gamma=10^{-4} \Omega_{0}$ in our numerical simulation. From Fig. 3(b), with the consideration of both the Rabi control error and the decoherence effect, we find that the SR-NGQC can significantly improve the robustness of quantum gates with the relative pulse deviation $|\beta|>0.01$.

On the other hand, one can find from Fig. 3(b) that the DG is better than NGQC and SR-NGQC for the relative pulse deviation $\beta \in[-0.01,0.01]$ since decoherence is the main factor in this case. In order to balance between the decoherence and the Rabi control errors, we plot the NOT gate fidelities' difference, $\Delta F=F_{G}-F_{D}\left(\Delta F=F_{N}-F_{D}\right)$ between the SR-NGQC (NGQC) and DG, with the decoherence parameter $\Gamma$ and the relative pulse deviation $\beta$, as shown in Fig. 3(d) [Fig. 3(c)]. We find that the best scheme in this case depends on the relative importance between the decoherence and the Rabi control error. An open question for further development is whether designing a hybrid SR-NGQC and DG scheme is optimal for quantum control. In addition, we 
clearly know that the NOT gate of the NGQC has no particular advantage compared to DG with both above errors, as shown in Fig. 3(c).

Conclusion and outlook. In conclusion, we have explained why the existing nonadiabatic geometric gates are so sensitive to the control errors, but also proposed the scheme of super-robust nonadiabatic geometric gates. Specifically, we have taken two examples in two- and three-level systems for realizing super-robust condition-based Abelian (non-Abelian) nonadiabatic geometric (holonomic) quantum gates, respectively. The theoretical and numerical results indicate that our scheme can significantly improve the gate performance compared to the existing geometric and standard dynamical schemes with the experimental parameters. Moreover, our scheme can also be extended to construct two-qubit geometric gates $[37,45,49,50]$ to realize a universal SR-based geometric gate set. In addition, this extensible approach of SR-based geometric gates can be applied to various physical platforms such as superconducting circuits, quantum dots, and trapped ions. For future work, it would be attractive for fault-tolerant quantum computation to combine a SR-based geometric scheme with the decoherence-free subspace (DFS) [26-28,68-70] encoding model (surface codes [71-75]) to further suppress the dephasing noises (local errors). In addition, it would be interesting to further optimize the magnetic field sensitivity and maximum field range of geometric-phase magnetometry [76] via our SR-NGQC scheme.

Acknowledgments. The authors thank Professor S.-L. Su for helpful discussions and improving this Letter. This work is supported by the Natural Science Foundation of Guangdong Province (Grant No. 2017B030308003), the Key R \& D Program of Guangdong Province (Grant No. 2018B030326001), the Science, Technology and Innovation Commission of Shenzhen Municipality (Grants No. JCYJ20170412152620376, No. JCYJ20170817105046702, and No. KYTDPT20181011104202253), the National Natural Science Foundation of China (Grants No. 11875160 and No. U1801661), the Economy, Trade and Information Commission of Shenzhen Municipality (Grant No. 201901161512), and Guangdong Provincial Key Laboratory (Grant No. 2019B121203002).
[1] M. V. Berry, Quantal phase factors accompanying adiabatic changes, Proc. R. Soc. London A 392, 45 (1984).

[2] Y. Aharonov and J. Anandan, Phase Change During a Cyclic Quantum Evolution, Phys. Rev. Lett. 58, 1593 (1987).

[3] P. Zanardi and M. Rasetti, Holonomic quantum computation, Phys. Lett. A 264, 94 (1999).

[4] J. Anandan, Non-adiabatic non-Abelian geometric phase, Phys. Lett. A 133, 171 (1988).

[5] E. Sjöqvist, Trend: A new phase in quantum computation, Physics 1, 35 (2008).

[6] S.-L. Zhu and P. Zanardi, Geometric quantum gates that are robust against stochastic control errors, Phys. Rev. A 72, 020301(R) (2005).

[7] S. Berger, M. Pechal, A. A. Abdumalikov, Jr., C. Eichler, L. Steffen, A. Fedorov, A. Wallraff, and S. Filipp, Exploring the effect of noise on the Berry phase, Phys. Rev. A 87, 060303(R) (2013).

[8] G. D. Chiara and G. M. Palma, Berry Phase for a Spin-1/2 Particle in a Classical Fluctuating Field, Phys. Rev. Lett. 91, 090404 (2003).

[9] P. J. Leek, J. M. Fink, A. Blais, R. Bianchetti, M. Göppl, J. M. Gambetta, D. I. Schuster, L. Frunzio, R. J. Schoelkopf, and A. Wallraff, Observation of Berry's phase in a solid state qubit, Science 318, 1889 (2007).

[10] S. Filipp, J. Klepp, Y. Hasegawa, C. Plonka-Spehr, U. Schmidt, P. Geltenbort, and H. Rauch, Experimental Demonstration of the Stability of Berry's Phase for a Spin-1/2 Particle, Phys. Rev. Lett. 102, 030404 (2009).

[11] F. Wilczek and A. Zee, Appearance of Gauge Structure in Simple Dynamical Systems, Phys. Rev. Lett. 52, 2111 (1984).

[12] J. A. Jones, V. Vedral, A. Ekert, and G. Castagnoli, Geometric quantum computation using nuclear magnetic resonance, Nature (London) 403, 869 (2000).

[13] H. Wu, E. M. Gauger, R. E. George, M. Möttönen, H. Riemann, N. V. Abrosimov, P. Becker, H.-J. Pohl, K. M. Itoh, M. L. W.
Thewalt, and J. J. L. Morton, Geometric phase gates with adiabatic control in electron spin resonance, Phys. Rev. A 87, 032326 (2013).

[14] Y.-Y. Huang, Y.-K. Wu, F. Wang, P.-Y. Hou, W.-B. Wang, W.G. Zhang, W.-Q. Lian, Y.-Q. Liu, H.-Y. Wang, H.-Y. Zhang et al., Experimental Realization of Robust Geometric Quantum Gates with Solid-State Spins, Phys. Rev. Lett. 122, 010503 (2019).

[15] L. M. Duan, J. I. Cirac, and P. Zoller, Geometric manipulation of trapped ions for quantum computation, Science 292, 1695 (2001).

[16] L.-A. Wu, P. Zanardi, and D. A. Lidar, Holonomic Quantum Computation in Decoherence-Free Subspaces, Phys. Rev. Lett. 95, 130501 (2005).

[17] X. B. Wang and M. Keiji, Nonadiabatic Conditional Geometric Phase Shift with NMR, Phys. Rev. Lett. 87, 097901 (2001).

[18] S.-L. Zhu and Z. D. Wang, Implementation of Universal Quantum Gates Based on Nonadiabatic Geometric Phases, Phys. Rev. Lett. 89, 097902 (2002).

[19] J. T. Thomas, M. Lababidi, and M. Tian, Robustness of singlequbit geometric gate against systematic error, Phys. Rev. A 84, 042335 (2011).

[20] P. Z. Zhao, X.-D. Cui, G. F. Xu, E. Sjöqvist, and D. M. Tong, Rydberg-atom-based scheme of non-adiabatic geometric quantum computation, Phys. Rev. A 96, 052316 (2017).

[21] K. Z. Li, P. Z. Zhao, and D. M. Tong, Approach to realizing non-adiabatic geometric gates with prescribed evolution paths, Phys. Rev. Research 2, 023295 (2020).

[22] T. Chen and Z.-Y. Xue, Non-Adiabatic Geometric Quantum Computation with Parametrically Tunable Coupling, Phys. Rev. Appl. 10, 054051 (2018).

[23] C. Zhang, T. Chen, S. Li and Z.-Y. Xue, High-fidelity geometric gate for silicon-based spin qubits, Phys. Rev. A 101, 052302 (2020). 
[24] B.-J. Liu, X.-K. Song, Z.-Y. Xue, X. Wang, and M.-H. Yung, Plug-and-Play Approach to Nonadiabatic Geometric Quantum Gates, Phys. Rev. Lett. 123, 100501 (2019).

[25] E. Sjöqvist, D. M. Tong, L. M. Andersson, B. Hessmo, M. Johansson, and K. Singh, Non-adiabatic holonomic quantum computation, New J. Phys. 14, 103035 (2012).

[26] G. F. Xu, J. Zhang, D. M. Tong, E. Sjöqvist, and L. C. Kwek, Nonadiabatic Holonomic Quantum Computation in Decoherence-free Subspaces, Phys. Rev. Lett. 109, 170501 (2012).

[27] Z.-Y. Xue, J. Zhou, and Z. D. Wang, Universal holonomic quantum gates in decoherence-free subspace on superconducting circuits, Phys. Rev. A 92, 022320 (2015).

[28] Z.-Y. Xue, F.-L. Gu, Z.-P. Hong, Z.-H. Yang, D.-W. Zhang, Y. $\mathrm{Hu}$, and J. Q. You, Non-Adiabatic Holonomic Quantum Computation with Dressed-State Qubits, Phys. Rev. Appl. 7, 054022 (2017).

[29] J. Zhou, B. J. Liu, Z. P. Hong, and Z. Y. Xue, Fast holonomic quantum computation based on solid-state spins with all-optical control, Sci. China-Phys. Mech. Astron. 61, 010312 (2018).

[30] Z.-P. Hong, B.-J. Liu, J.-Q. Cai, X.-D. Zhang, Y. Hu, Z. D. Wang, and Z.-Y. Xue, Implementing universal non-adiabatic holonomic quantum gates with transmons, Phys. Rev. A 97, 022332 (2018).

[31] V. Azimi Mousolou, Electric non-adiabatic geometric entangling gates on spin qubits, Phys. Rev. A 96, 012307 (2017).

[32] P. Z. Zhao, K. Z. Li, G. F. Xu, and D. M. Tong, General approach for constructing Hamiltonians for non-adiabatic holonomic quantum computation, Phys. Rev. A 101, 062306 (2020).

[33] M. Johansson, E. Sjöqvist, L. M. Andersson, M. Ericsson, B. Hessmo, K. Singh, and D. M. Tong, Robustness of nonadiabatic holonomic gates, Phys. Rev. A 86, 062322 (2012).

[34] S. B. Zheng, C. P. Yang, and F. Nori, Comparison of the sensitivity to systematic errors between nonadiabatic non-Abelian geometric gates and their dynamical counterparts, Phys. Rev. A 93, 032313 (2016).

[35] N. Ramberg and E. Sjöqvist, Environment-Assisted Holonomic Quantum Maps, Phys. Rev. Lett. 122, 140501 (2019).

[36] J. Jing, C.-H. Lam, and L.-A. Wu, Non-Abelian holonomic transformation in the presence of classical noise, Phys. Rev. A 95, 012334 (2017).

[37] Y. Xu, Z. Hua, Tao Chen, X. Pan, X. Li, J. Han, W. Cai, Y. Ma, H. Wang, Y. P. Song, Z.-Y. Xue, and L. Sun, Experimental Implementation of Universal Non-Adiabatic Geometric Quantum Gates in a Superconducting Circuit, Phys. Rev. Lett. 124, 230503 (2020).

[38] P. Z. Zhao, Z. Dong, Z. Zhang, G. Guo, D. M. Tong, and Y. Yin, Experimental realization of non-adiabatic geometric gates with a superconducting xmon qubit, Sci. China-Phys. Mech. Astron. 64, 250362 (2021).

[39] C. Song, S.-B. Zheng, P. Zhang, K. Xu, L. Zhang, Q. Guo, W. Liu, D. Xu, H. Deng, K. Huang et al., Continuous-variable geometric phase and its manipulation for quantum computation in a superconducting circuit, Nat. Commun. 8, 1061 (2017).

[40] T. Yan, B.-J. Liu, K. Xu, C. Song, S. Liu, Z. Zhang, H. Deng, Z. Yan, H. Rong, M.-H. Yung, Y. Chen, and D. Yu, Experimental Realization of Non-Adiabatic Shortcut to Non-Abelian Geometric Gates, Phys. Rev. Lett. 122, 080501 (2019).

[41] Z. Han, Y. Dong, B. Liu, X. Yang, S. Song, L. Qiu, D. Li, J. Chu, W. Zheng, J. Xu, T. Huang, Z. Wang, X. Yu,
X. Tan, D. Lan, M.-H. Yung, and Y. Yu, Experimental realization of universal time-optimal non-abelian geometric gates, arXiv:2004.10364.

[42] A. A. Abdumalikov, J. M. Fink, K. Juliusson, M. Pechal, S. Berger, A. Wallraff, and S. Filipp, Experimental realization of non-Abelian non-adiabatic geometric gates, Nature (London) 496, 482 (2013).

[43] Y. Xu, W. Cai, Y. Ma, X. Mu, L. Hu, T. Chen, H. Wang, Y. P. Song, Z.-Y. Xue, Z.-Q. Yin, and L. Sun, Single-Loop Realization of Arbitrary Nonadiabatic Holonomic Single Qubit Quantum Gates in a Superconducting Circuit, Phys. Rev. Lett. 121, 110501 (2018).

[44] S. Danilin, A. Vepsäläinen, and G. S. Paraoanu, Experimental state control by fast non-Abelian holonomic gates with a superconducting qutrit, Phys. Scr. 93, 055101 (2018).

[45] D. J. Egger, M. Ganzhorn, G. Salis, A. Fuhrer, P. Muller, P. K. Barkoutsos, N. Moll, I. Tavernelli, and S. Filipp, Entanglement Generation in Superconducting Qubits Using Holonomic Operations, Phys. Rev. Appl. 11, 014017 (2019).

[46] G. Feng, G. Xu, and G. Long, Experimental Realization of NonAdiabatic Holonomic Quantum Computation, Phys. Rev. Lett. 110, 190501 (2013).

[47] H. Li, L. Yang, and G. Long, Experimental realization of singleshot non-adiabatic holonomic gates in nuclear spins, Sci. China: Phys., Mech. Astron. 60, 080311 (2017).

[48] Z. Zhu, T. Chen, X. Yang, J. Bian, Z.-Y. Xue, and X. Peng, Single-Loop and Composite-Loop Realization of non-adiabatic Holonomic Quantum Gates in a Decoherence-Free Subspace, Phys. Rev. Appl. 12, 024024 (2019).

[49] C. Zu, W.-B. Wang, L. He, W.-G. Zhang, C.-Y. Dai, F. Wang, and L.-M. Duan, Experimental realization of universal geometric quantum gates with solid-state spins, Nature (London) 514, 72 (2014).

[50] K. Nagata, K. Kuramitani, Y. Sekiguchi, and H. Kosaka, Universal holonomic quantum gates over geometric spin qubits with polarized microwaves, Nat. Commun. 9, 3227 (2018).

[51] S. Arroyo-Camejo, A. Lazariev, S. W. Hell, and G. Balasubramanian, Room temperature high-fidelity holonomic single-qubit gate on a solid-state spin, Nat. Commun. 5, 4870 (2014).

[52] Y. Sekiguchi, N. Niikura, R. Kuroiwa, H. Kano, and H. Kosaka, Optical holonomic single quantum gates with a geometric spin under a zero field, Nat. Photon. 11, 309 (2017).

[53] B. B. Zhou, P. C. Jerger, V. O. Shkolnikov, F. Joseph Heremans, G. Burkard, and D. D. Awschalom, Holonomic Quantum Control by Coherent Optical Excitation in Diamond, Phys. Rev. Lett. 119, 140503 (2017).

[54] N. Ishida, T. Nakamura, T. Tanaka, S. Mishima, H. Kano, R. Kuroiwa, Y. Sekiguchi, and H. Kosaka, Universal holonomic single quantum gates over a geometric spin with phasemodulated polarized light, Opt. Lett. 43, 2380 (2018).

[55] X. Rong, J. Geng, F. Shi, Y. Liu, K. Xu, W. Ma, F. Kong, Z. Jiang, Y. Wu, and J. Du, Experimental fault-tolerant universal quantum gates with solid-state spins under ambient conditions, Nat. Commun. 6, 8748 (2015).

[56] X. Wang, L. S. Bishop, J. P. Kestner, E. Barnes, K. Sun, and S. D. Sarma, Composite pulses for robust universal control of singlet-triplet qubits, Nat. Commun. 3, 997 (2012).

[57] H. Ribeiro, A. Baksic, and A. A. Clerk, Systematic Magnus- 
Based Approach for Suppressing Leakage and Non-Adiabatic Errors in Quantum Dynamics, Phys. Rev. X 7, 011021 (2017).

[58] W. Magnus, On the exponential solution of differential equations for a linear operator, Commun. Pure Appl. Math. 7, 649 (1954).

[59] S. Blanes, F. Casas, J. A. Oteo, and J. Ros, The magnus expansion and some of its applications, Phys. Rep. 470, 151 (2009).

[60] A. Souza, G. A. Alvarez, and D. Suter, Robust dynamical decoupling, Philos. Trans. R. Soc. A 370, 4748 (2012).

[61] G. T. Genov, D. Schraft, N. V. Vitanov, and T. Halfmann, Arbitrarily Accurate Pulse Sequences for Robust Dynamical Decoupling, Phys. Rev. Lett. 118, 133202 (2017).

[62] M. Kolodrubetz, D. Sels, P. Mehta, and A. Polkovnikov, Geometry and non-adiabatic response in quantum and classical systems, Phys. Rep. 697, 1 (2017).

[63] See Supplemental Material at http://link.aps.org/supplemental/ 10.1103/PhysRevResearch.3.L032066 for a discussion of the choices of time-dependent auxiliary states and the parameters of a NOT gate for SR-NHQC and SR-NGQC, which includes Refs. [20,24-26,43].

[64] G. Lindblad, On the generators of quantum dynamical semigroups, Commun. Math. Phys. 48, 119 (1976).

[65] I. Buluta, S. Ashhab, and F. Nori, Natural and artificial atoms for quantum computation, Rep. Prog. Phys. 74, 104401 (2011).

[66] Z. Chen, J. Kelly, C. Quintana, R. Barends, B. Campbell, Y. Chen, B. Chiaro, A. Dunsworth, A. G. Fowler, E. Lucero et al., Measuring and Suppressing Quantum State Leakage in a Superconducting Qubit, Phys. Rev. Lett. 116, 020501 (2016).

[67] P. Krantz, M. Kjaergaard, F. Yan, T. P. Orlando, S.
Gustavsson, and W. D. Oliver, A quantum engineer's guide to superconducting qubits, Appl. Phys. Rev. 6, 021318 (2019)

[68] X. K. Song, H. Zhang, Q. Ai, J. Qiu, and F. G. Deng, Shortcuts to adiabatic holonomic quantum computation in decoherencefree subspace with transitionless quantum driving algorithm, New J. Phys. 18, 023001 (2016).

[69] Z.-T. Liang, Y.-X. Du, W. Huang, Z.-Y. Xue, and H. Yan, nonadiabatic holonomic quantum computation in decoherence-free subspaces with trapped ions, Phys. Rev. A 89, 062312 (2014).

[70] X.-L. Feng, C. Wu, H. Sun, and C. H. Oh, Geometric Entangling Gates in Decoherence-Free Subspaces with Minimal Requirements, Phys. Rev. Lett. 103, 200501 (2009).

[71] S. B. Bravyi and A. Y. Kitaev, Quantum codes on a lattice with boundary, arXiv:quant-ph/9811052.

[72] E. Dennis, A. Y. Kitaev, A. Landahl, and J. Preskill, Topological quantum memory, J. Math. Phys. 43, 4452 (2002).

[73] A. G. Fowler, M. Mariantoni, J. M. Martinis, and A. N. Cleland, Surface codes: Towards practical large-scale quantum computation, Phys. Rev. A 86, 032324 (2012).

[74] J. Zhang, S. J. Devitt, J. Q. You, and F. Nori, Holonomic surface codes for fault-tolerant quantum computation, Phys. Rev. A 97, 022335 (2018).

[75] C. Wu, Y. Wang, X.-L. Feng, and J.-L. Chen, Holonomic Quantum Computation in Surface Codes, Phys. Rev. Appl. 13, 014055 (2020).

[76] K. Arai, J. Lee, C. Belthangady, D. R. Glenn, H. Zhang, and R. L. Walsworth, Geometric phase magnetometry using a solidstate spin, Nat. Commun. 9, 4996 (2018). 